\title{
Os Autores brasileiros não citam os autores brasileiros: Nada mudou desde 1994*
}

\section{Brazilian authors don't cite Brazilian authors: Nothing has changed since 1994}

\author{
Vincenzo Giordano ${ }^{1,2(0)}$ Juliana Lyra $^{3}$ João Artur Bonadiman ${ }^{4}$ Osvandré Lech ${ }^{5}$ (i)
}

${ }^{1}$ Serviço de Ortopedia e Traumatologia Prof. Nova Monteiro, Hospital Municipal Miguel Couto, Rio de Janeiro, RJ, Brasil

${ }^{2}$ Clínica São Vicente, Rede D’or São Luiz, Rio de Janeiro, RJ, Brasil

3 Programa de Ortopedia e Traumatologia, Serviço de Ortopedia e Traumatologia Prof. Nova Monteiro, Hospital Municipal Miguel Couto, Rio de Janeiro, RJ, Brasil

${ }^{4}$ Instituto de Ortopedia e Traumatologia, Hospital São Vicente de Paulo, Passo Fundo, RS, Brasil

${ }^{5}$ Serviço de Cirurgia do Ombro e Cotovelo, Instituto de Ortopedia e Traumatologia, Hospital São Vicente de Paulo, Passo Fundo, RS, Brasil

Rev Bras Ortop 2021;56(2):154-160.
Endereço para correspondência Vincenzo Giordano, Rua Mário Ribeiro, $117 / 2^{\circ}$ andar, Leblon, 22430-160, Rio de Janeiro, RJ, Brasil (e-mail:v_giordano@me.com).

\section{Resumo \\ Palavras-chave \\ - artigo de revista \\ - fator de impacto de revistas \\ - revisão da pesquisa por pares \\ - base de dados}

Objetivo Observar o perfil de autocitações da Revista Brasileira de Ortopedia (Rev Bras Ortop) e de citações deste periódico em outras revistas médicas de ortopedia de conteúdo geral ou específico de uma determinada área de conhecimento da especialidade.

Métodos Trata-se de estudo observacional transversal da frequência de autocitações e citações da Rev Bras Ortop em outros cinco periódicos médicos de ortopedia de diferentes países, todas publicadas em língua inglesa. Foram analisados os 15 últimos artigos publicados em 2020 em cada uma das seis revistas estudadas. As referências usadas em cada um delas foi avaliada para identificação do periódico em que foram publicadas originalmente. A distribuição de frequência dos quatro principais periódicos citados, sua posição e o percentual relativo ao total de citações foram observados e registrados em cada uma das seis revistas. O número de vezes em que a Rev Bras Ortop foi citada em cada um dos periódicos estrangeiros selecionados foi avaliado por meio de suas frequências absoluta e relativa.

\footnotetext{
* Trabalho desenvolvido pelo Serviço de Ortopedia e Traumatologia Prof. Nova Monteiro, Hospital Municipal Miguel Couto, Rio de Janeiro, RJ e Hospital São Vicente de Paulo, Instituto de Ortopedia e Traumatologia, Passo Fundo, RS, Brasil.
}

recebido

07 de Fevereiro de 2021

aceito

19 de Fevereiro de 2021
DOI https://doi.org/

10.1055/s-0041-1728702. ISSN 0102-3616.
(C) 2021. Sociedade Brasileira de Ortopedia e Traumatologia. All rights reserved.

This is an open access article published by Thieme under the terms of the Creative Commons Attribution-NonDerivative-NonCommercial-License, permitting copying and reproduction so long as the original work is given appropriate credit. Contents may not be used for commercial purposes, or adapted, remixed, transformed or built upon. (https://creativecommons.org/ licenses/by-nc-nd/4.0/)

Thieme Revinter Publicações Ltda., Rua do Matoso 170, Rio de Janeiro, RJ, CEP 20270-135, Brazil 


\begin{abstract}

\section{Keywords}

- journal article

- journal impact factor

- peer review

- research

- database

Objective To outline the profile of self-citations of the Revista Brasileira de Ortopedia (Rev Bras Ortop) and citations of this journal in other medical orthopaedic journals with general or specific content in a knowledge area of the specialty.

Methods This is an observational cross-sectional study of the frequency of selfcitations and citations from Rev Bras Ortop in five other medical orthopaedic journals from different countries, all published in English. The last 15 articles published in 2020 in each of the six journals were analyzed. The references used in each of them were evaluated to identify the journal in which they were originally published. The frequency of distribution of the four main journals cited, their position, and the relative percentage to the total number of citations were observed and recorded in each of the six journals. The number of times that the Rev Bras Ortop was cited in each of the selected foreign journals was assessed using its absolute and relative frequencies.

Results The total number of citations evaluated in this study was 2,527 (ranging from 386 to 486 per magazine). Rev Bras Ortop showed a low rate of self-citation (2.6\%), being the sixth journal cited in the journal itself (10 out of a total of 386 references). Moreover, Rev Bras Ortop was not mentioned in any of the other five medical journals included in the study (absolute frequency 0 , relative frequency 0 ).

Conclusion Rev Bras Ortop has a low reference of itself, with a self-citation rate of $2.6 \%$ in the period studied, showing that the Brazilian orthopaedic surgeons do not mention the Brazilian orthopaedic surgeon who publishes in the journal. We suggest the elaboration and implementation of strong strategies to improve the journal's visibility in the world academic-scientific scenario. In addition, it is essential that Brazilian orthopaedic surgeons understand this reality and assist directly and effectively to change this scenario.
\end{abstract}

Resultados O total de citações avaliadas neste estudo foi de 2527 (variando de 386 a 486 por revista). A Rev Bras Ortop apresentou baixa taxa de autocitação (2,6\%), sendo citada na própria revista na sexta posição (10 de um total de 386 referências). No período estudado, a Rev Bras Ortop não foi citada em nenhum dos outros cinco periódicos médicos incluídos no estudo (frequência absoluta 0 , frequência relativa 0 ). Conclusão Observou-se que a Rev Bras Ortop apresenta baixa referência de si própria, com taxa de autocitação de $2,6 \%$ no período estudado, mostrando que de fato o ortopedista brasileiro não cita o ortopedista brasileiro que publica na revista. Sugerimos a elaboração e a implementação de estratégias fortes de melhora da visibilidade do periódico no cenário acadêmico-científico mundial. Além disso, é fundamental que os ortopedistas brasileiros entendam esta realidade e auxiliem direta e efetivamente em sua mudança.

\section{Introdução}

Pesquisar, escrever e divulgar os achados de uma investigação científica são meios importantes de comunicação no meio acadêmico. ${ }^{1}$ De modo geral, o papel da publicação tem função tanto acadêmica, de informar e comunicar os resultados da pesquisa, quanto profissional, democraticamente fornecendo uma área estruturada para o debate dos achados do estudo. ${ }^{1,2}$ Nesse contexto, a principal função das revistas médicas é transmitir informações que melhorem a assistência à saúde, publicando artigos científicos que enfoquem assuntos de grande relevância na prática de saúde. ${ }^{3}$ No entanto, apesar do crescente número de publicações científicas, permitindo alcançar um público mais amplo de leitores, os autores enfrentam um desafio sem precedentes ao selecionar qual o periódico ideal para publicar sua pesquisa. $^{3-5}$

Nessa difícil tomada de decisão, o fator de impacto (FI) da revista tem sido um dos fatores mais amplamente utilizados para avaliar sua qualidade, importância e penetração no meio acadêmico. ${ }^{6,7}$ O Fl é calculado dividindo o número de citações de um periódico no Journal Citation Reports (JCR) em um ano pelo número total de artigos publicados na mesma disciplina no periódico avaliado nos dois anos anteriores. ${ }^{8}$ Alguns autores têm sugerido a escolha de periódicos científicos de 
propriedade de uma associação de profissionais de saúde ou de publicações que permitam acesso livre ao leitor, potencialmente aumentando a visibilidade do estudo. ${ }^{9,10}$

No Brasil, a Revista Brasileira de Ortopedia (Rev Bras Ortop) é o órgão de publicação científica da Sociedade Brasileira de Ortopedia e Traumatologia (SBOT). ${ }^{11}$ A revista não recebe dinheiro de financiamento de agências de fomento, sendo totalmente suportada pela SBOT, sem que haja a cobrança de taxa para submissão e publicação de seus artigos. Desde 2009 é indexada nos bancos de dados PubMed, PubMed Central, Scopus, SciELO (Scientific Eletronic Library On-Line) e LILACS (Literatura Latino-americana em Ciências da Saúde), garantindo boa visibilidade no cenário ortopédico mundial. Apesar disso, Lech, em editorial publicado na própria revista em 1994, chamou a atenção para o fato de que os autores nacionais não citam os autores nacionais, demonstrado pela baixa taxa de autocitação dentro da revista. ${ }^{12}$ Dentre as causas apontadas, destacam-se a necessidade de usar referências internacionais para "conferir veracidade" ao estudo e os fenômenos do "terceiro-mundismo" e da "concorrência implícita" entre os autores.

Curiosamente, apesar de ter aumentado sua exposição em bancos de dados de excelência em pesquisa médica, desde 1994 pouco mudou neste cenário pobre de citações da própria revista. ${ }^{13}$ Mas será que os autores brasileiros de fato citam menos a revista de sua associação profissional do que os autores estrangeiros a de suas sociedades ou associações médicas? Nossa hipótese é que pouco mudou desde 1994, mantendo-se baixo o número de autocitações da revista. O objetivo deste estudo é observar o perfil de autocitações da Rev Bras Ortop e de citações deste periódico em outras revistas médicas de ortopedia.

\section{Material e Métodos}

Trata-se de estudo observacional transversal da frequência de autocitações e citações da Rev Bras Ortop em outros periódicos médicos de ortopedia. Além da Rev Bras Ortop, foram escolhidas outras quatro revistas de abrangência geral dentro da especialidade (The Journal of Bone $\mathcal{E}$ Joint Surg American [J Bone Joint Surg Am], Bone \& Joint Journal [Bone Joint J], Acta Orthopaedica et Traumatologica Turcica [Acta Orthop Traumatol Turc] e Der Unfallchirurgie [Unfallchirurg]) e uma revista específica de uma área de conhecimento (Journal of Shoulder $\mathcal{E}$ Elbow Surgery [J Shoulder Elbow Surg]).

O J Bone Joint Surg Am é uma revista médica quinzenal revisada por pares e publicada pela The Journal of Bone and Joint Surgery, Inc. (Massachusetts, EUA). Está indexada no PubMed, Scopus, CrossRef, Portico e Web of Science, com FI 2019 de 4,57. ${ }^{14}$ O Bone Joint J, anteriormente conhecido como The Journal of Bone EJ Joint Surgery British, é uma revista médica mensal revisada por pares e publicada pela The British Editorial Society of Bone \& Joint Surgery (Londres, Reino Unido). Está indexada no PubMed, com FI 2019 de 4,30. ${ }^{15}$ A Acta Orthop Traumatol Turc é o jornal oficial da Associação Turca de Ortopedia e Traumatologia (Türk Ortopedi ve Travmatoloji Derneğ $\mathrm{i}$ - TOTDER) e da Sociedade Turca de Ortopedia e Traumatologia (Türk Ortopedi ve Travmatoloji Birliği Derneği - TOTBID). É um periódico científico de acesso aberto, revisado por pares, publicado bimestralmente em língua inglesa. Está indexada no Science Citation Index Expanded, PubMed, PubMed Central, Scopus, DOAJ, Index Copernicus e TUBITAK ULAKBIM TR Index, com FI 2019 de $1,21 .{ }^{16}$ A Unfallchirurg é a revista médica oficial da Sociedade Alemã de Cirurgia de Trauma (Deutschen Gesellschaft für Unfallchirurgie), de periodicidade mensal, oferecendo alguns artigos de acesso aberto e outros somente por assinatura. Os artigos são revisados por pares e publicados originalmente em língua alemã, com resumo em língua inglesa. Está indexada no Science Citation Index (SCI) Expanded, PubMed, EMBASE e Scopus, com FI 2019 de $0,67 .{ }^{17}$ O J Shoulder Elbow Surg é a publicação oficial de diversas sociedades médicas de especialidade, incluindo a Sociedad Latinoamericana de Hombro y Codo. Sua periodicidade é mensal e seus artigos são revisados por pares. Oferece artigos de acesso aberto e outros somente por assinatura. Está indexada no PubMed, EMBASE e Scopus, com FI 2019 de $2,81 .{ }^{18}$ A - Tabela 1 traz as informações das revistas selecionadas.

Foram selecionados os 15 últimos artigos publicados em 2020 em cada uma das seis revistas. Todas as referências foram avaliadas para identificação do periódico em que foram publicadas originalmente. A distribuição de frequência pontual dos quatro principais periódicos citados, sua posição e o percentual relativo ao total de citações foram observados em cada uma das revistas. O número de vezes em que a Rev Bras Ortop foi citada em cada um dos periódicos selecionados foi avaliado por meio de suas frequências absoluta e relativa.

\section{Resultados}

O total de citações neste estudo foi de 2527, variando de 386 (Rev Bras Ortop) a 486 (J Shoulder Elbow Surg) por revista. Três revistas apresentaram alta frequência e três revistas apresentaram baixa frequência de citações de si próprias. Observou-se que o J Shoulder Elbow Surg, o Bone Joint J e o J Bone Joint Surg Am apresentam referência de si próprias em primeiro lugar, com $22,2 \%, 13,7 \%$ e $11,9 \%$ das citações, respectivamente, em relação ao total pesquisado da revista. A Rev Bras Ortop, a Acta Orthop Traumatol Turc e a Unfallchirurg apresentaram baixa referência de si próprias, com 2,6\%, 1,0\% e 2,2\% das citações, respectivamente, em relação ao total pesquisado da revista. A Acta Orthop Traumatol Turc citou a si própria quatro vezes em um total de 391 referências e a Unfallchirurg 10 vezes de um total de 456 referências. A Rev Bras Ortop aparece citada na própria revista na sexta posição (10 de um total de 386 referências usadas no período do estudo), entretanto não foi citada em nenhum dos outros cinco periódicos médicos incluídos no estudo (frequência absoluta 0 , frequência relativa 0 ).

A - Tabela 2 traz as seis revistas de ortopedia analisadas no estudo, com suas quatro principais citações, além do número de vezes que a Rev Bras Ortop foi citada em cada um dos periódicos.

\section{Discussão}

Observou-se que, de uma forma geral, existe baixa frequência de citações da Rev Bras Ortop tanto na própria revista quanto 
Tabela 1 Informações das revistas médicas de ortopedia usadas no estudo

\begin{tabular}{|c|c|c|c|c|}
\hline Revista Principal & Quem publica & Periodicidade & Indexação & FI 2019 \\
\hline Rev Bras Ortop & SBOT & Mensal & $\begin{array}{l}\text { PubMed, PubMed Central, } \\
\text { Scopus, SciELO e LILACS }\end{array}$ & 0,69 \\
\hline J Bone Joint Surg Am & $\begin{array}{l}\text { The Journal of Bone and } \\
\text { Joint Surgery, Inc. }\end{array}$ & Quinzenal & $\begin{array}{l}\text { PubMed, Scopus, CrossRef, } \\
\text { Portico e Web of Science }\end{array}$ & 4,57 \\
\hline Bone Joint J & $\begin{array}{l}\text { The British Editorial Society } \\
\text { of Bone \& Joint Surgery }\end{array}$ & Mensal & PubMed & 4,30 \\
\hline $\begin{array}{l}\text { Acta Orthop } \\
\text { Traumatol Turc }\end{array}$ & TOTDER / TOTBID & Bimestral & $\begin{array}{l}\text { Science Citation Index Expanded, } \\
\text { PubMed, PubMed Central, Scopus, } \\
\text { DOAJ, Index Copernicus e } \\
\text { TUBITAK ULAKBIM TR Index }\end{array}$ & 1,21 \\
\hline Unfallchirurg & $\begin{array}{l}\text { Deutschen Gesellschaft } \\
\text { für Unfallchirurgie }\end{array}$ & Mensal & $\begin{array}{l}\text { Science Citation Index Expanded, } \\
\text { PubMed, EMBASE e Scopus }\end{array}$ & 0,67 \\
\hline J Shoulder Elbow Surg & $\begin{array}{l}\text { diversas sociedades médicas } \\
\text { de especialidade } \#\end{array}$ & Mensal & PubMed, EMBASE e Scopus & 2,81 \\
\hline
\end{tabular}

Abbreviations: Fl, fator de impacto.

Fonte: SOT Prof. Nova Monteiro-Hospital Municipal Miguel Couto / IOT Passo Fundo, 2021.

Legendas:

Rev Bras Ortop - Revista Brasileira de Ortopedia

J Bone Joint Surg Am - The Journal of Bone \& Joint Surgery American

Bone Joint J - The Bone \& Joint Journal

Acta Orthop Traumatol Turc - Acta Orthopaedica et Traumatologica Turcica

Unfallchirurg - Der Unfallchirurgie

J Shoulder Elbow Surg - The Journal of Shoulder \& Elbow Surgery

SBOT - Sociedade Brasileira de Ortopedia e Traumatologia

TOTDER - Türk Ortopedi ve Travmatoloji Derneği

TOTBID - Türk Ortopedi ve Travmatoloji Birliği Derneği

\# - American Shoulder and Elbow Surgeons, European Society for Surgery of Shoulder and Elbow, Japan Shoulder Society, Shoulder and Elbow Society of Australia, Sociedad Latinoamericana de Hombro y Codo, South African Shoulder and Elbow Surgeons, Asian Shoulder Association, Korean Shoulder and Elbow Society, International Congress of Shoulder and Elbow Surgery e American Society of Shoulder and Elbow Therapists.

nos demais periódicos pesquisados neste estudo. Na avaliação dos 15 últimos artigos publicados na Rev Bras Ortop em 2020, somente 10 foram da própria revista, ao passo que nos outros cinco periódicos incluídos no estudo a revista não foi citada nenhuma vez. A magnitude do problema é enorme, haja vista ser o principal veículo de divulgação de evidências científicas dos ortopedistas brasileiros. Embora não tenha sido nosso objetivo avaliar as razões que levam o ortopedista brasileiro a citar tão pouco sua própria revista, nossos achados sugerem a necessidade de elaboração e execução de estratégias de incentivo à citação de sua revista científica.

Existem duas formas de lidar com esta triste realidade. Uma delas é, de modo simplista, buscar um "culpado," seja este o fenômeno "terceiro-mundista," a busca por um "bom" periódico estrangeiro ou o próprio ortopedista brasileiro cioso de "sua" descoberta. No entanto, deve-se ter em mente que não existe uma lista confiável de periódicos bons e ruins, e que o compartilhamento de evidências por meio de publicações científicas contribui muito ao minimizar a importância das fronteiras e ao contribuir para a utilização de evidências no âmbito da saúde global. ${ }^{1,5}$ Além disso, não há conhecimento "local" ou "muito específico" que não interesse a qualquer revista médica que busque qualidade e transparência. ${ }^{19}$

A outra maneira de lidar com o problema da baixa autocitação da Rev Bras Ortop é melhorar sua reputação, o que inclui a indexação em mais bases de dados bibliográficos, a percepção de "prestígio" do periódico e o aumento do seu
FI, amplamente utilizado na seleção de qual periódico enviar o estudo científico. ${ }^{2,3,20,21}$ Interessante notar que dos três periódicos com maior número de autocitações, o menor FI 2019 é 2,81, ${ }^{18}$ enquanto o maior FI 2019 das revistas com menor número de autocitações é $1,21 .^{16}$ Atualmente o FI 2019 da Rev Bras Ortop é 0,69. ${ }^{11}$ Curiosamente, dos três periódicos de menor autocitação, todos são de órgãos oficias de suas sociedades de especialidade médica. Apesar do aumento potencial da visibilidade do estudo entre um número maior de profissionais da área quando este é publicado no periódico de sua própria associação médica profissional, ${ }^{9,10}$ Morley e Urquhart ${ }^{2}$ observaram que esse vínculo foi apontado como de baixa importância entre profissionais de um hospital universitário do National Health System do Reino Unido.

Embora o FI seja importante critério de escolha de qual periódico submeter um estudo científico, diversos autores têm apontado limitações em seu uso, especialmente por manipulação e aplicação incorretas de autocitações. ${ }^{7,8,22}$ Ademais, sua utilidade não se estende a artigos individuais, sugerindo que não há objetivamente nenhuma correlação entre a frequência de citação de um artigo individual e o FI de determinada publicação. ${ }^{19,22,23}$ Finalmente, é importante notar que o FI não reflete a qualidade e a transparência no processo da revisão por pares à qual um periódico sujeita seus artigos. ${ }^{3,5,24}$ No estudo de Morley e Urquhart, ${ }^{2}$ a revisão por pares foi considerado um fator muito importante na 
Tabela 2 Principais citações nas seis revistas de ortopedia incluídas no estudo

\begin{tabular}{|c|c|c|c|c|}
\hline Revista Principal & Citações principais & Posição & $\begin{array}{l}\text { Frequência } \\
\text { absoluta }\end{array}$ & $\begin{array}{l}\text { Frequência relativa } \\
\text { ao total de citações (\%) }\end{array}$ \\
\hline \multicolumn{5}{|l|}{ Rev Bras Ortop } \\
\hline $\begin{array}{l}\text { Total de citações } \\
\text { pesquisadas: } 386\end{array}$ & $\begin{array}{l}\text { Knee Surg Sports Traumatol Arthrosc } \\
\text { Am J Sports Med } \\
\text { J Bone Joint Surg Am } \\
\text { Spine } \\
\text { Rev Bras Ortop - a própria } \\
\text { TOTAL PARCIAL }\end{array}$ & $\begin{array}{l}1^{\mathrm{a}} \\
2^{\mathrm{a}} \\
3^{\mathrm{a}} \\
4^{\mathrm{a}} \\
6^{\mathrm{a}}\end{array}$ & $\begin{array}{l}90 \\
29 \\
28 \\
20 \\
10 \\
177\end{array}$ & $\begin{array}{l}23,3 \\
7,5 \\
7,3 \\
5,2 \\
2,6 \\
45,9\end{array}$ \\
\hline \multicolumn{5}{|c|}{ J Bone Joint Surg Am } \\
\hline $\begin{array}{l}\text { Total de citações } \\
\text { pesquisadas: } 420\end{array}$ & $\begin{array}{l}\text { J Bone Joint Surg Am - a própria } \\
\text { Clin Orthop Rel Res } \\
\text { Am J Sports Med } \\
\text { J Arthroplasty } \\
\text { Rev Bras Ortop } \\
\text { TOTAL PARCIAL }\end{array}$ & $\begin{array}{l}1^{\mathrm{a}} \\
2^{\mathrm{a}} \\
3^{\mathrm{a}} \\
4^{\mathrm{a}} \\
\mathrm{N} / \mathrm{C}\end{array}$ & $\begin{array}{l}50 \\
28 \\
22 \\
17 \\
0 \\
117\end{array}$ & $\begin{array}{l}11,9 \\
6,7 \\
5,2 \\
4,0 \\
0 \\
27,9\end{array}$ \\
\hline \multicolumn{5}{|l|}{ Bone Joint J } \\
\hline $\begin{array}{l}\text { Total de citações } \\
\text { pesquisadas: } 388\end{array}$ & $\begin{array}{l}\text { Bone Joint J - a própria } \\
\text { J Bone Joint Surg Am } \\
\text { Spine } \\
\text { Clin Orthop Rel Res } \\
\text { Rev Bras Ortop } \\
\text { TOTAL PARCIAL }\end{array}$ & $\begin{array}{l}1^{\mathrm{a}} \\
2^{\mathrm{a}} \\
3^{\mathrm{a}} \\
4^{\mathrm{a}} \\
\mathrm{N} / \mathrm{C}\end{array}$ & $\begin{array}{l}53 \\
44 \\
34 \\
16 \\
0 \\
147\end{array}$ & $\begin{array}{l}13,7 \\
11,3 \\
8,8 \\
4,1 \\
0 \\
37,9\end{array}$ \\
\hline \multicolumn{5}{|c|}{ Acta Orthop Traumatol Turc } \\
\hline $\begin{array}{l}\text { Total de citações } \\
\text { pesquisadas: } 391\end{array}$ & $\begin{array}{l}\text { J Bone Joint Surg Am } \\
\text { Clin Orthop Rel Res } \\
\text { J Hand Surg Am } \\
\text { J Shoulder Elbow Surg } \\
\text { Acta Orthop Traumatol Turc - a própria } \\
\text { Rev Bras Ortop } \\
\text { TOTAL PARCIAL }\end{array}$ & $\begin{array}{l}1^{a} \\
2^{a} \\
3^{a} \\
4^{a} \\
19^{a} \\
N / C\end{array}$ & $\begin{array}{l}24 \\
23 \\
17 \\
15 \\
4 \\
0 \\
83\end{array}$ & $\begin{array}{l}6,1 \\
5,9 \\
4,3 \\
3,8 \\
1,0 \\
0 \\
21,2\end{array}$ \\
\hline \multicolumn{5}{|l|}{ Unfallchirurg } \\
\hline $\begin{array}{l}\text { Total de citações } \\
\text { pesquisadas: } 456\end{array}$ & $\begin{array}{l}\text { Am J Sports Med } \\
\text { J Bone Joint Surg Am } \\
\text { J Shoulder Elbow Surg } \\
\text { Bone Joint J } \\
\text { Unfallchirurg - a própria } \\
\text { Rev Bras Ortop } \\
\text { TOTAL PARCIAL }\end{array}$ & $\begin{array}{l}1^{\mathrm{a}} \\
2^{\mathrm{a}} \\
2^{\mathrm{a}} \\
4^{\mathrm{a}} \\
6^{\mathrm{a}} \\
\mathrm{N} / \mathrm{C}\end{array}$ & $\begin{array}{l}33 \\
27 \\
27 \\
15 \\
10 \\
0 \\
112 \\
\end{array}$ & $\begin{array}{l}7,2 \\
5,9 \\
5,9 \\
3,3 \\
2,2 \\
0 \\
24,6\end{array}$ \\
\hline \multicolumn{5}{|c|}{ J Shoulder Elbow Surg } \\
\hline $\begin{array}{l}\text { Total de citações } \\
\text { pesquisadas: } 486\end{array}$ & $\begin{array}{l}\text { J Shoulder Elbow Surg - a própria } \\
\text { J Bone Joint Surg Am } \\
\text { Arthroscopy } \\
\text { Am J Sports Med } \\
\text { Rev Bras Ortop } \\
\text { TOTAL PARCIAL }\end{array}$ & $\begin{array}{l}1^{\mathrm{a}} \\
2^{\mathrm{a}} \\
2^{\mathrm{a}} \\
4^{\mathrm{a}} \\
\mathrm{N} / \mathrm{C}\end{array}$ & $\begin{array}{l}108 \\
49 \\
19 \\
18 \\
0 \\
194\end{array}$ & $\begin{array}{l}22,2 \\
10,1 \\
3,9 \\
3,70 \\
39,9\end{array}$ \\
\hline
\end{tabular}

Abbreviation: N/C, não citado.

Fonte: SOT Prof. Nova Monteiro-Hospital Municipal Miguel Couto / IOT Passo Fundo, 2021.

Legendas:

Rev Bras Ortop - Revista Brasileira de Ortopedia

J Bone Joint Surg Am - The Journal of Bone \& Joint Surgery American

Bone Joint J - The Bone \& Joint Journal

Acta Orthop Traumatol Turc - Acta Orthopaedica et Traumatologica Turcica

Unfallchirurg - Der Unfallchirurgie

J Shoulder Elbow Surg - The Journal of Shoulder \& Elbow Surgery

Knee Surg Sports Traumatol Arthrosc - Knee Surgery, Sports Traumatology, Arthoscopy

Am J Sports Med - The American Journal of Sports Medicine

Clin Orthop Rel Res - Clinical Orthopaedics and Related Research

J Arthroplasty - The Journal of Arthroplasty

J Hand Surg Am - The Journal of Hand Surgery American volume 
escolha do periódico para publicação. Periódicos respeitáveis devem divulgar totalmente seu processo de revisão por pares no conteúdo impresso ou em sua página oficial da Internet, e seus revisores devem entender a importância de seu trabalho na legitimação da publicação. ${ }^{5,24-26}$ A falta de revisão por pares leva a práticas antiéticas, como plágio, publicação de dados falsificados não científicos e práticas clínicas inseguras. ${ }^{25}$ Em 2018, mais de 42.000 periódicos acadêmicos revisados por pares ativos foram publicados, com um crescimento acelerado de mais de $5 \%$ nos últimos anos. ${ }^{27}$

A influência dos estudos da América Latina na literatura ortopédica tem sido limitada, com pequeno número de publicações realizadas na região. ${ }^{28,29} \mathrm{Em} 2014$, os países latino-americanos produziram apenas $1 \%$ de todos os artigos ortopédicos publicados. ${ }^{29}$ Dos 50 artigos mais citados, 20 eram de autores brasileiros, mas nenhum periódico nacional foi usado como referência. Nesse contexto, o que é necessário para que os ortopedistas brasileiros reconheçam a importância da Rev Bras Ortop e a escolham para publicar seus estudos? Mais ainda, como motivar a citação aos autores nacionais que publicam na revista? Compreender as características que tornam os artigos citados por outros cientistas pode ajudar pesquisadores, instituições e governos a promover pesquisas de qualidade que poderiam se tornar mais influentes para a comunidade científica ortopédica internacional. ${ }^{29}$

Este desafio parece não ser de exclusividade da Rev Bras Ortop. Em nosso estudo, observamos que dos quatro periódicos publicados por suas associações profissionais de especialidade, somente o Bone Joint J autocitou-se em primeiro lugar. Certamente esse achado reflete a diversidade de assuntos cobertos pelo Bone Joint J, mas também suas reputação e credibilidade construídas ao longo de anos. Chomsky-Higgins et al. ${ }^{28}$ ressaltaram a necessidade de políticas fortes de incentivo à produção científica, como apoio a projetos multicêntricos e estudos clínicos que beneficiem a população local, estímulo a residentes e jovens ortopedistas a produzir pesquisas de qualidade e melhoria da infraestrutura hospitalar. Desse modo, como entidade profissional e educativa maior da ortopedia brasileira, a SBOT precisa fomentar o treinamento e obter o financiamento necessário para desenvolver dados nacionais que possam ser publicados em sua própria revista, aumentando a visibilidade das pesquisas e dos pesquisadores brasileiros no cenário mundial. Em paralelo, existe a necessidade desta e de outras associações médicas profissionais agirem junto a instituições governamentais, como a CAPES, de modo a diminuir a pressão burocrática, caracterizada pela elevação arbitrária dos níveis de corte para fins de financiamento, que leva autores nacionais a tentarem publicar seus manuscritos em revistas com maior FI em seu campo de pesquisa, ao invés de buscar um periódico brasileiro, como a Rev Bras Ortop. ${ }^{30}$

Dentre as limitações do estudo, destacam-se principalmente o curto período de coleta dos dados (últimos 15 artigos publicados em 2020) e a falta de investigação das razões que levam o ortopedista brasileiro a citar tão pouco sua própria revista. Por tratar-se de estudo observacional transversal, examinamos a taxa de autocitação em um determinado momento, avaliando a força da relação entre o fator exposição e variáveis bibliométricas levadas em consideração em periódicos científicos, tais como FI, revisão por pares e principal fonte editorial. Sabidamente estudos transversais não possuem uma dimensão temporal inerente, pois verificam a prevalência do fator exposição em tempo presente. ${ }^{31}$ Assim, observamos que a Rev Bras Ortop mostrou baixa taxa de autocitação, sugerindo a necessidade da implantação de estratégias pontuais para reversão do cenário atual. Entender as razões da baixa taxa de autocitação é fundamental, mas o fato de não ter sido este o foco do presente estudo, não inviabiliza a tomada de ações que melhorem a visibilidade da principal publicação científica da SBOT.

\section{Conclusão}

Observou-se que a Revista Brasileira de Ortopedia apresenta baixa taxa de autocitação, mostrando que o ortopedista brasileiro não cita o ortopedista brasileiro que publica na revista. Sugerimos a elaboração e a implementação de estratégias fortes de melhora da visibilidade do periódico no cenário acadêmico-científico mundial. É fundamental que os ortopedistas brasileiros entendam esta realidade e auxiliem direta e efetivamente em sua mudança.

Conflito de Interesses

Os autores declaram não haver conflito de interesses

\section{Referências}

1 Asnake M. The importance of scientific publication in the development of public health. Cien Saude Colet 2015;20(07): 1972-1973

2 Morley P, Urquhart C. Publishing trends in medical journal literature: the views of medical staff in an NHS teaching hospital in the United Kingdom. Health Info Libr J 2004;21 (02):121-124

3 Hudzik B. What makes a good medical journal great? CMAJ 2016; 188(07):531

4 Garba S, Ahmed A, Mai A, Makama G, Odigie V. Proliferations of scientific medical journals: a burden or a blessing. Oman Med J 2010;25(04):311-314

5 Suiter AM, Sarli CC. Selecting a journal for publication: criteria to consider. Mo Med 2019;116(06):461-465

6 Not-so-deep impact. Nature 2005;435(7045):1003-1004

7 Sundaram K, Warren J, Anis HK, Klika AK, Piuzzi NS. Publication integrity in orthopaedic journals: the self-citation in orthopaedic research (SCOR) threshold. Eur J Orthop Surg Traumatol 2020;30 (04):629-635

8 Garfield E. The history and meaning of the journal impact factor. JAMA 2006;295(01):90-93

9 Björk BC, Solomon D. Open access versus subscription journals: a comparison of scientific impact. BMC Med 2012;10:73

10 Suzuki K, Edelson A, Iversen LL, Hausmann L, Schulz JB, Turner AJ. A learned society's perspective on publishing. J Neurochem 2016; 139(Suppl 2):17-23

11 Revista Brasileira de Ortopedia. [Last accessed in January 2021] from https://www.scielo.br/revistas/rbort/iaboutj.htm

12 Lech 0 . Os autores brasileiros não citam os autores brasileiros. [Editorial]Rev Bras Ortop 1994;29(09):

13 Lech 0 . Autores nacionais não citam os autores nacionais-parte 2. Comunicação Pessoal; 2020 
14 The Journal of Bone \& Joint Surgery. [Last accessed in January 2021] from https://www.ovid.com/product-details.11933.html

15 The Bone \& Joint Journal. [Last accessed in January 2021] from: https://online.boneandjoint.org.uk/journal/bjj

16 Acta Orthopaedica et Traumatologica Túrcica. [Last accessed in January 2021] from https://www.aott.org.tr/

17 Der Unfallchirurg. [Last accessed in January 2021] from https:// www.springer.com/journal/113

18 Journal of Shoulder and Elbow Surgery. Last accessed in January 2021 from https://www.jshoulderelbow.org/

19 Marusić M Why physicians should publish, how easy it is, and how important it is in clinical work. Arch Oncol 2003;11(02):59-64

20 Balhara YP. Indexed journal: What does it mean? Lung India 2012; 29(02):193

21 Seglen PO. Citation rates and journal impact factors are not suitable for evaluation of research. Acta Orthop Scand 1998;69 (03):224-229

22 Kurmis AP. Understanding the limitations of the journal impact factor. J Bone Joint Surg Am 2003;85(12):2449-2454

23 Whitehouse GH. Impact factors: facts and myths. Eur Radiol 2002; 12(04):715-717
24 Chloros GD, Giannoudis VP, Giannoudis PV. Peer-reviewing in surgical journals: revolutionize or perish? Ann Surg 2021

25 Rupp M, Anastasopoulou L, Wintermeyer E, Malhaan D, El Khassawna T, Heiss C. Predatory journals: a major threat in orthopaedic research. Int Orthop 2019;43(03):509-517

26 Smith R. The trouble with medical journals. J R Soc Med 2006;99 (03):115-119

27 Wilkinson J. How to find the right journal for your research (using actual data). [Last accessed in January 2021] from: https://clarivate. com/webofsciencegroup/article/how-to-find-the-right-journal-foryour-research-using-actual-data/

28 Chomsky-Higgins K, Miclau TA, Mackechnie MC, et al. Barriers to clinical research in Latin America. Front Public Health 2017;5:57

29 Urrutia J, Zamora T, Prada C. The fifty most cited Latin-American articles in the orthopaedic literature. Int Orthop 2014;38(08): 1723-1729

30 Metze K. Bureaucrats, researchers, editors, and the impact factor: a vicious circle that is detrimental to science. Clinics (Sao Paulo) 2010;65(10):937-940

31 Song JW, Chung KC. Observational studies: cohort and casecontrol studies. Plast Reconstr Surg 2010;126(06):2234-2242 